\title{
Estimation of Biomass Potential in Pirgulu Innate Zone
}

\author{
Ulviyya Mammadova
}

Department of Soils Ecology and Bonitation, Institute of Soilscience and Agrochemistry of ANAS, Baki, AZ1073, Azerbaijan

\begin{abstract}
Biomass potential is one of the very interesting energy sources in the nature. In the paper the estimation of biomass potential from the cattle husbandry was given. While calculating the resource all populations amount within the families living in the region were taken into account. Also the difference between the biomass quantity from cow and buffalo has been appreciated. At the same time the total biogas reserve obtained from the biomass collected in the personal husbandries was defined. Finally the ecological and economical aspects of biogas has been validated.
\end{abstract}

Keywords Biomass Potential, Biogas Reserve, Cattle Husbandry, Personal Farms

\section{Introduction}

Multiple works are being carried out in alternative energy field over all the world at present. Thus in several world countries, solar, wind, thermal water, hydroenergy, biomass, wave and other renewable energy potentials have been calculated[3,14,16] and these energy resources are being widely used now. For both energy supply and ecological problems solution renewable enrgy sources application is profitable from ecological facet per each country.Particularly in the countries or zones where cattle-breeding sector has developed perfectly biomass potential is able to be utilized in order to generate bigass energy $[5,10,12]$. The biogas can be used in any field of the life and also in different buildings to provide heating need. Biogasapplication practise exists and proved itself in the international level. Biogas energy plants as other reneable energy equipments are to be exploitated individually and in centralized form. Combined application of biogas lets good condition generation to utilize the current resources effectively in preventing environment from being polluted and reducing amount of the ecological questions formation[6,8]. At the same time forets safety and decreasing forest wood hewing biogas is one of the most beneficial cleen energy sources. Forests protection and reforestation are very actual matters now. It should be taken into considration that the initial reason of forest cutting comes from the heat demand of the population. Finally in order to delay fuelwood hewing $[7,2,4]$ beside solar and wind energy potentials biomass potential is worthy source to generate biogas[15]. In the contact with woodlands for the purpose of supplying settlements with bogas energy the total amount of biomass reserve (wet brick) ought to be determined and calculated.

\footnotetext{
* Corresponding author:

uf_samadova@yahoo.com (Ulviyya Mammadova)

Published online at http://journal.sapub.org/ep

Copyright $(C 2012$ Scientific \& Academic Publishing. All Rights Reserved
}

During organizing biogas generation population amount in the region, heat energy necessity and collected biomass reserve should be taken into account beforehand. Therefore around the investigation area, in thesettlements and villages surrounding Pirgulu region total amount of the polulation was determined statistically. Because while calculating biomass from the cattle-breeding husbandries this factor is important, at least. Potential determination is too necessary in order to validate biomass application.

In formating the energy sector initially energy stocks and reserves are to be calculated[9,16]. Whilst utilizing the energy stocks economical and ecological aspects have to be appreciated for vindicating the energy potential

exploitation in the life and different fields of industry sectors. Really almost in the former SSR period cattle -breeding husbandry was developed and extended in the country. But the waste of this industry field are partly used in cultivation of soils by fertilizing. The rest wates haven't used anyway. Historically cattle-breeding wastes were utilized by the population as the energy source in dry brick $[1,16]$ form especially there where finding energy sources was impossible in mountainous and foothil regions. But biogas generation didn't come to their mind. Lately biogas usage leads to biomass utilization from cattle-breeding farms. Practically in German[3], Turkey[8] and other countries biogass generation is realized and they supply the local population with biogas source[11]. One of the companies who is busy with biogas energy plants purchase is russian one by name "Kiska". This company proposed his energy plants to be instolate in some mountainous village on the mountainous zone. In this zone energy transportation and pipline building are difficult because of the natural condition andrelief state $[5,13]$. Some of the houses agreed to use these plants with working biomass resource. Effective result was obtained. So this proves that application and exploitation of the existing bimass potential in the regions of the country is possible and profitable. That's the time to carry out this work 
in the connecting zones with the forests where hugely forest fuelwood is used for providing energy need. So the biomass potential has to be calculated and determined for validating the potential usage in advance.

\section{Object and Methods of the Study}

While selecting investigation zone the renewable energy potential reserve and location of the villages surrounding woodlands have been taken into consideration. One of such innate zone is Pirgulu region which is rich with wealthy flora, fauna on the Great Caucasus mountain in Shamakhi region. Pirgulu innate zone has a lot of renewable energy resources. Due to the objective of the investigation amount of families, populations in the settlements in the region have been determined and tabled below. Here not only the villages surrounding Pirgulu, also other villages in the region which carry the fuelwood from Pirgulu woodland were considered in calculations.

Also for the purpose of biogas generation and heat energy supply average amount of cattle (cow and buffalo) in the personal husbandry of one settlement on the total quantity has been determined. In the investigation zone in villages amount of biomass obtained from the personal cattle -breeding farms was revealed beforehand. Also the daily biomass (wet brick) weight from the cattle was defined and used in the calculation. During studying it was validated that amount of cattle-breeding in surrounding zone of Pirgulu consists of 1338,25 buffalos, 2676,5 cows, total index is 4014,75 . Averagely weight of biomass obtained from coq is $8-12 \mathrm{~kg}$ (sometimes $10 \mathrm{~kg}$ ), weight of biomass obtained from buffalo is $15-20 \mathrm{~kg}$ (sometimes $17,5 \mathrm{~kg}$ ). Due to the indicates above at table 2, average daily amount of biomass from cattle-breeding (cow and buffalo) has been determined and given at the table.

On indexes obtained after calculations realized in villages surrounding Pirgulu biogas amount generated from biomass collected in the personal cattle-breeding for each settlement has been calculated, then defined. Averagely $1 \mathrm{~m}^{3}$ biogas is generated from $10 \mathrm{~kg}$ biomass, by this way in Avakhil village biomass obtained from cow in cattle-breeding farm per a person has been calculated. The result was $44 \mathrm{~kg}$. but the daily biomass reserve averagely for Avakhil $44 \times 490=21$ $560 \mathrm{~kg}$ cow biomass. So, during a day from this cow biomass averagely $21560 \div 10=2156 \mathrm{~m}^{3}$ biogas is generated.

In the very village biomass amount collected from buffalo per a person averagely is $47,3 \mathrm{~kg}$. While taking into account all the population $47,3 \times 490=23177 \mathrm{~kg}$, so biogas generated from buffalo biomass will be $23177 \div 10=2317,7 \mathrm{~m}^{3}$.

Generally biogas quantity generated from cow and buffalo cattle-breeding biomass $2156+23177=25333 \mathrm{~m}^{3}$. by this way according to the population amount for other villagesaverage daily biomass quantity and biogas capacity generated from this biomass were determined. The results of thecalculation have been given at the following table correspondingly.

Table 1. Amount of the population living in villages surrounding Pirgulu State Nature Preserve

\begin{tabular}{|c|c|c|c|c|c|c|}
\hline № & Villages & Family amount & $\begin{array}{l}\text { Population } \\
\text { amount }\end{array}$ & $\begin{array}{l}\text { Average family } \\
\text { member }\end{array}$ & $\begin{array}{c}\text { Buffalo amount } \\
\text { per a person }\end{array}$ & $\begin{array}{r}\text { Cow amount } \\
\text { per a person }\end{array}$ \\
\hline 1 & Avakhil & 99 & 490 & 4,9 & 2,7 & 4,4 \\
\hline 2 & Chabani & 76 & 387 & 6 & 3,4 & 4,9 \\
\hline 3 & Chukhuryurd & 794 & 1048 & 1,32 & 1,3 & 2,5 \\
\hline 4 & Dedegunesh & 52 & 198 & 3,8 & 4,7 & 4,5 \\
\hline 5 & Demirchi & 102 & 582 & 5,7 & 2,3 & 4,5 \\
\hline 6 & Ahmadli & 123 & 502 & 5 & 2,7 & 3,3 \\
\hline 7 & Angakharan & 196 & 856 & 4,4 & 1,6 & 3,2 \\
\hline 8 & Archiman & 139 & 856 & 4,4 & 1,6 & 3,2 \\
\hline 9 & Hajili & 73 & 365 & 5 & 1 & 4,6 \\
\hline 10 & I Chagan & 44 & 365 & 5 & 3,6 & 3,3 \\
\hline 11 & II Chagan & 36 & 149 & 4,2 & 4,3 & 4,7 \\
\hline 12 & Kechmeddin & 51 & 211 & 4,1 & 2,9 & 5,8 \\
\hline 13 & Galaderesi & 36 & 143 & 4 & 2,9 & 6,1 \\
\hline 14 & Galeybugurd & 192 & 801 & 4,2 & 2,6 & 5,2 \\
\hline 15 & Gizmeydan & 258 & 1114 & 4,3 & 4,4 & 3,8 \\
\hline 16 & Gonagkend & 166 & 829 & 5 & 3,4 & 4,9 \\
\hline 17 & Gurdtepe & 139 & 804 & 6 & 1,7 & 3,5 \\
\hline 18 & Meysari & 162 & 690 & 4,2 & 1,2 & 2,4 \\
\hline 19 & Malham & 414 & 1392 & 3,4 & 1,6 & 3,4 \\
\hline 20 & Nagarakhana & 212 & 864 & 4 & 1,7 & 3,3 \\
\hline 21 & Pirbayli & 38 & 184 & 5 & 1,9 & 3,9 \\
\hline 22 & Pirgulu settlement & 6 & 41 & 6,8 & $x$ & $\times$ \\
\hline 23 & Safali & 31 & 134 & 4,3 & 1 & 1,9 \\
\hline 24 & Sis & 79 & 348 & 4,4 & 1,9 & 3,9 \\
\hline 25 & Talishnuru & 77 & 317 & 4,1 & 1 & 1,9 \\
\hline 26 & Yeni Gizmeydan & 23 & 95 & 4,1 & 1,5 & 3 \\
\hline 27 & Zarat Kheyberi & 40 & 233 & 5,8 & 3,3 & 6 \\
\hline sum: & 27 villages (one settlement) & 3658 & 13546 & \begin{tabular}{l|l} 
Average & 4,5 \\
\end{tabular} & 3 & 4,2 \\
\hline
\end{tabular}


Table 2. Biomass amount in personal cattle farms in villages surrounding Pirgulu State Nature Preserve

\begin{tabular}{|c|c|c|c|c|}
\hline № & Villages & $\begin{array}{l}\text { Average daily buffalo biomass } \\
\text { amount, } \mathrm{kg}\end{array}$ & $\begin{array}{c}\text { Average daily cow biomass } \\
\text { amount, } \mathrm{kg}\end{array}$ & $\begin{array}{l}\text { Average daily biomass } \\
\text { amount from cattle, } \mathrm{kg}\end{array}$ \\
\hline 1 & Avakhil & 47,3 & 44 & 45,7 \\
\hline 2 & Chabani & 59,5 & 49 & 54,25 \\
\hline 3 & Chukhuryurd & 22,8 & 25 & 23,9 \\
\hline 4 & Dedegunesh & 82,3 & 45 & 63,65 \\
\hline 5 & Demirchi & 40,3 & 45 & 42,65 \\
\hline 6 & Ahmadli & 47,3 & 33 & 40,15 \\
\hline 7 & Angakharan & 28 & 32 & 30 \\
\hline 8 & Archiman & 28 & 32 & 30 \\
\hline 9 & Hajili & 17,5 & 46 & 31,75 \\
\hline 10 & I Chagan & 63 & 33 & 48 \\
\hline 11 & II Chagan & 75,3 & 47 & 61,15 \\
\hline 12 & Kechmeddin & 50,8 & 58 & 54,4 \\
\hline 13 & Galaderesi & 50,8 & 61 & 55,9 \\
\hline 14 & Galeybugurd & 45,5 & 52 & 97,5 \\
\hline 15 & Gizmeydan & 77 & 38 & 57,5 \\
\hline 16 & Gonagkend & 29,8 & 49 & 78,8 \\
\hline 17 & Gurdtepe & 12,8 & 35 & 23,9 \\
\hline 18 & Meysari & 21 & 24 & 22,5 \\
\hline 19 & Malham & 28 & 34 & 31 \\
\hline 20 & Nagarakhana & 29,8 & 33 & 62,8 \\
\hline 21 & Pirbayli & 33,3 & 39 & 36,15 \\
\hline 22 & Pirgulu settlement & $x$ & $x$ & $x$ \\
\hline 23 & Safali & 17,5 & 19 & 18,25 \\
\hline 24 & Sis & 26,3 & 30 & 28,15 \\
\hline 25 & Talishnuru & 57,8 & 60 & 58,9 \\
\hline 26 & Yeni Gizmeydan & 38,5 & 42 & 40,25 \\
\hline 27 & Zarat Kheyberi & 85,8 & 54 & 69,9 \\
\hline sum: & 27 villages (one settlement) & 43 & 40,7 & 46,5 \\
\hline
\end{tabular}

Table 3. Average index of biomass and biogas potential obtained in the settlements on populations' amount

\begin{tabular}{|c|c|c|c|c|c|}
\hline № & Villages & $\begin{array}{l}\text { Buffalo biomass due to } \\
\text { population amount }(\mathrm{kg})\end{array}$ & $\begin{array}{l}\text { Cow biomass due to } \\
\text { population amount }(\mathrm{kg})\end{array}$ & $\begin{array}{l}\text { Biogas from } \\
\text { buffalo }\left(\mathrm{M}^{3}\right)\end{array}$ & $\begin{array}{c}\text { Biogas from cow } \\
\left(M^{3}\right)\end{array}$ \\
\hline 1 & Avakhil & 23177 & 21560 & 2318 & 2156 \\
\hline 2 & Chabani & 23026,5 & 18963 & 2303 & 1897 \\
\hline 3 & Chukhuryurd & 23894,4 & 26200 & 2390 & 2620 \\
\hline 4 & Dedegunesh & 16295,4 & 8910 & 1630 & 891 \\
\hline 5 & Demirchi & 23454,6 & 26190 & 2346 & 2619 \\
\hline 6 & Ahmadli & 23744,6 & 16566 & 2375 & 1657 \\
\hline 7 & Angakharan & 23968 & 27392 & 2397 & 2740 \\
\hline 8 & Archiman & 10237,5 & 26910 & 1024 & 2691 \\
\hline 9 & Hajili & 22995 & 12045 & 2300 & 1205 \\
\hline 10 & I Chagan & 13855,2 & 8648 & 1386 & 865 \\
\hline 11 & II Chagan & 7569,2 & 9089 & 757 & 909 \\
\hline 12 & Kechmeddin & 16247 & 8018 & 1625 & 802 \\
\hline 13 & Galaderesi & 4261,4 & 7007 & 427 & 701 \\
\hline 14 & Galeybugurd & 10252,8 & 28035 & 1026 & 2804 \\
\hline 15 & Gizmeydan & 23394 & 26736 & 2340 & 2674 \\
\hline 16 & Gonagkend & 23212 & 28186 & 2322 & 2819 \\
\hline 17 & Gurdtepe & 23959,2 & 26532 & 2396 & 2654 \\
\hline 18 & Meysari & 22977 & 26910 & 2298 & 2691 \\
\hline 19 & Malham & 24360 & 26448 & 2436 & 2645 \\
\hline 20 & Nagarakhana & 22723,2 & 25920 & 2273 & 2592 \\
\hline 21 & Pirbayli & 10635,2 & 11040 & 10636 & 1104 \\
\hline 22 & Pirgulu settlement & $\times$ & $\times$ & $\times$ & $\times$ \\
\hline 23 & Safali & 11497,2 & 7236 & 1150 & 724 \\
\hline 24 & Sis & 23142 & 24360 & 2313 & 2436 \\
\hline 25 & Talishnuru & 9985,5 & 19971 & 9986 & 1998 \\
\hline 26 & Yeni Gizmeydan & 5652,5 & 4085 & 5653 & 409 \\
\hline 27 & Zarat Kheyberi & 23253,4 & 23300 & 23254 & 2330 \\
\hline sum: & 27 villages (one settlement) & 467769,8 & 496257 & 91361 & 49633 \\
\hline
\end{tabular}


Table 4. Biomass and biogas generation from cow farms in villages surrounding Pirgulu State Nature Preserve

\begin{tabular}{|c|c|c|c|c|c|c|c|}
\hline № & Villages & $\begin{array}{c}\text { Cow amount } \\
\text { per a person } \\
\text { (head) }\end{array}$ & $\begin{array}{l}\text { Average daily } \\
\text { Biomass kg }\end{array}$ & $\begin{array}{l}\text { average annual } \\
\text { biomass } \mathrm{kg}\end{array}$ & $\begin{array}{c}\text { Average annual } \\
\text { biogas generation } \\
\mathrm{m}^{3}\end{array}$ & $\begin{array}{l}\text { Average monthly } \\
\text { biogas generation } \\
\mathrm{m}^{3}\end{array}$ & $\begin{array}{l}\text { Demand } \\
\text { provision } \\
\text { reserve \% }\end{array}$ \\
\hline 1 & Avakhil & 4,4 & 88 & 31680 & 3168 & 264 & 58 \\
\hline 2 & Chabani & 4,9 & 98 & 35280 & 3528 & 294 & 65 \\
\hline 3 & Chukhuryurd & 2,5 & 50 & 18000 & 1800 & 150 & 33 \\
\hline 4 & Dedegunesh & 4,5 & 90 & 32400 & 3240 & 270 & 60 \\
\hline 5 & Demirchi & 4,5 & 90 & 32400 & 3240 & 270 & 60 \\
\hline 6 & Ahmadli & 3,3 & 66 & 23760 & 2376 & 198 & 44 \\
\hline 7 & Angakharan & 3,2 & 64 & 23040 & 2304 & 192 & 42 \\
\hline 8 & Archiman & 3,2 & 64 & 23040 & 2304 & 192 & 42 \\
\hline 9 & Hajili & 4,6 & 92 & 33120 & 3312 & 276 & 61 \\
\hline 10 & I Chagan & 3,3 & 66 & 23760 & 2376 & 198 & 44 \\
\hline 11 & II Chagan & 4,7 & 94 & 33840 & 3384 & 282 & 62 \\
\hline 12 & Kechmeddin & 5,8 & 116 & 41760 & 4176 & 348 & 77 \\
\hline 13 & Galaderesi & 6,1 & 122 & 43920 & 4392 & 366 & 81 \\
\hline 14 & Galeybugurd & 5,2 & 104 & 37440 & 3744 & 312 & 69 \\
\hline 15 & Gizmeydan & 3,8 & 76 & 27360 & 2736 & 228 & 50 \\
\hline 16 & Gonagkend & 4,9 & 98 & 35280 & 3528 & 294 & 65 \\
\hline 17 & Gurdtepe & 3,5 & 70 & 25200 & 2520 & 210 & 46 \\
\hline 18 & Meysari & 2,4 & 48 & 17280 & 1728 & 144 & 32 \\
\hline 19 & Malham & 3,4 & 68 & 24480 & 2448 & 204 & 45 \\
\hline 20 & Nagarakhana & 3,3 & 66 & 23760 & 2376 & 198 & 44 \\
\hline 21 & Pirbayli & 3,9 & 78 & 28080 & 2808 & 234 & 52 \\
\hline 22 & Pirgulu settlement & $x$ & $x$ & $x$ & $x$ & $x$ & $x$ \\
\hline 23 & Safali & 1,9 & 38 & 13680 & 1368 & 114 & 25 \\
\hline 24 & Sis & 3,9 & 78 & 28080 & 2808 & 234 & 52 \\
\hline 25 & Talishnuru & 1,9 & 38 & 13680 & 1368 & 114 & 25 \\
\hline 26 & Yeni Gizmeydan & 3 & 60 & 21600 & 2160 & 180 & 40 \\
\hline 27 & Zarat Kheyberi & 6 & 120 & 43200 & 4320 & 360 & 80 \\
\hline sum: & $\begin{array}{l}27 \text { villages (one } \\
\text { settlement) }\end{array}$ & average & 4,2 & 78,5 & 2827,4 & 235,6 & 52 \\
\hline
\end{tabular}

Table 5. Biomass and biogas reserves from buffalo farms in villages surrounding Pirgulu State Nature Preserve

\begin{tabular}{|c|c|c|c|c|c|c|c|c|}
\hline № & Villages & $\begin{array}{r}\text { Buffalo amour } \\
\text { a person }\end{array}$ & per & $\begin{array}{c}\text { Average daily } \\
\text { biomass, } \\
\text { kg }\end{array}$ & $\begin{array}{c}\text { Annual } \\
\text { biomass index } \\
\mathrm{kg} \\
\end{array}$ & $\begin{array}{c}\text { Annual biogas } \\
\text { generation } \\
\mathrm{m}^{3}\end{array}$ & $\begin{array}{c}\text { Average monthly } \\
\text { biogas generation } \\
\mathrm{m}^{3}\end{array}$ & $\begin{array}{c}\text { Demand } \\
\text { provision reserve } \\
\% \\
\end{array}$ \\
\hline 1 & Avakhil & 2,7 & & 67,5 & 24300 & 2430 & 202,5 & 45 \\
\hline 2 & Chabani & 3,4 & & 85 & 30600 & 3060 & 255 & 56 \\
\hline 3 & Chukhuryurd & 1,3 & & 32,5 & 11700 & 1170 & 97,5 & 21 \\
\hline 4 & Dedegunesh & 4,7 & & 117,5 & 42300 & 4230 & 352,5 & 78 \\
\hline 5 & Demirchi & 2,3 & & 57,5 & 20700 & 2070 & 172,5 & 38 \\
\hline 6 & Ahmadli & 2,7 & & 67,5 & 24300 & 2430 & 202,5 & 45 \\
\hline 7 & Angakharan & 1,6 & & 40 & 14400 & 1440 & 120 & 26 \\
\hline 8 & Archiman & 1,6 & & 40 & 14400 & 1440 & 120 & 26 \\
\hline 9 & Hajili & 1 & & 25 & 9000 & 900 & 75 & 16 \\
\hline 10 & I Chagan & 3,6 & & 90 & 32400 & 3240 & 270 & 60 \\
\hline 11 & II Chagan & 4,3 & & 107,5 & 38700 & 3870 & 322,5 & 71 \\
\hline 12 & Kechmeddin & 2,9 & & 72,5 & 26100 & 2610 & 217,5 & 48 \\
\hline 13 & Galaderesi & 2,9 & & 72,5 & 26100 & 2610 & 217,5 & 48 \\
\hline 14 & Galeybugurd & 2,6 & & 65 & 23400 & 2340 & 195 & 43 \\
\hline 15 & Gizmeydan & 4,4 & & 110 & 39600 & 3960 & 330 & 73 \\
\hline 16 & Gonagkend & 3,4 & & 85 & 30600 & 3060 & 255 & 56 \\
\hline 17 & Gurdtepe & 1,7 & & 42,5 & 15300 & 1530 & 127,5 & 28 \\
\hline 18 & Meysari & 1,2 & & 30 & 10800 & 1080 & 90 & 20 \\
\hline 19 & Malham & 1,6 & & 40 & 14400 & 1440 & 120 & 26 \\
\hline 20 & Nagarakhana & 1,7 & & 42,5 & 15300 & 1530 & 127,5 & 28 \\
\hline 21 & Pirbayli & 1,9 & & 47,5 & 17100 & 1710 & 142,5 & 31 \\
\hline 22 & Pirgulu settlement & $x$ & & $x$ & $x$ & $x$ & $x$ & $x$ \\
\hline 23 & Safali & 1 & & 25 & 9000 & 900 & 75 & 16 \\
\hline 24 & Sis & 1,9 & & 47,5 & 17100 & 1710 & 142,5 & 31 \\
\hline 25 & Talishnuru & 1 & & 25 & 9000 & 900 & 75 & 16 \\
\hline 26 & Yeni Gizmeydan & 1,5 & & 37,5 & 13500 & 1350 & 112,5 & 25 \\
\hline 27 & Zarat Kheyberi & 3,3 & & 82,5 & 29700 & 2970 & 247,5 & 55 \\
\hline sum: & $\begin{array}{l}27 \text { villages (one } \\
\text { settlement) }\end{array}$ & average & 3 & 60 & 21530,8 & 2154 & 179,5 & 39,5 \\
\hline
\end{tabular}


Table 6. Total biogas reserv from both husbandry in the surrounding villages

\begin{tabular}{|c|c|c|c|c|}
\hline № & Biomass potential in the villages & $\begin{array}{c}\text { Biogas from } \\
\text { cow husbandry } \\
\% \\
\end{array}$ & $\begin{array}{c}\text { Biogas from } \\
\text { buffalo husbandry } \\
\% \\
\end{array}$ & $\begin{array}{c}\text { Total average } \\
\text { biogas } \\
\% \\
\end{array}$ \\
\hline 1 & $\begin{array}{l}\text { Avakhil village with } 490 \text { peoples, } 2,7 \text { buffalos, } 4,4 \text { cows per a } \\
\text { person, average total biomass is } 22368,5 \mathrm{~kg} \text { on cattle-breeding }\end{array}$ & 58 & 45 & 52 \\
\hline 2 & $\begin{array}{l}\text { Chabani village with } 387 \text { peoples, } 3,4 \text { buffalos, } 4,9 \text { cows per a } \\
\text { person, average total biomass is } 20994,8 \mathrm{~kg} \text { on cattle-breeding }\end{array}$ & 65 & 56 & 61 \\
\hline 3 & $\begin{array}{c}\text { Chukhuryurd village with } 1048 \text { peoples, } 1,3 \text { buffalos, } 2,5 \text { cows per } \\
\text { a person, average total biomass is } 25047,2 \mathrm{~kg} \text { on cattle farm }\end{array}$ & 33 & 21 & 27 \\
\hline 4 & $\begin{array}{l}\text { Dedegunesh village with } 198 \text { peoples, } 4,7 \text { buffalos, } 4,7 \text { cows per a } \\
\text { person, average total biomass is } 25047,2 \mathrm{~kg} \text { on cattle-breeding }\end{array}$ & 60 & 78 & 69 \\
\hline 5 & $\begin{array}{l}\text { Demirchi village with } 582 \text { peoples, } 2,3 \text { buffalos, } 4,5 \text { cows per a } \\
\text { person, average total biomass is } 24822,3 \mathrm{~kg} \text { on cattle-breeding }\end{array}$ & 60 & 38 & 49 \\
\hline 6 & $\begin{array}{l}\text { Ahmedli village with } 502 \text { peoples, } 2,7 \text { buffalos, } 3,3 \text { cows per a } \\
\text { person, average total biomass is } 32566,45 \mathrm{~kg} \text { on cattle-breeding }\end{array}$ & 44 & 45 & 45 \\
\hline 7 & $\begin{array}{l}\text { Angekharan village with } 856 \text { peoples, } 1,6 \text { buffalos, } 3,2 \text { cows per } \\
\text { a person, average total biomass is } 25680 \mathrm{~kg} \text { on cattle-breeding }\end{array}$ & 42 & 26 & 34 \\
\hline 8 & $\begin{array}{l}\text { Archiman village with } 856 \text { peoples, } 1,6 \text { buffalos, } 3,2 \text { cows per a } \\
\text { person, average total biomass is } 18573,75 \mathrm{~kg} \text { on cattle-breeding }\end{array}$ & 42 & 26 & 34 \\
\hline 9 & $\begin{array}{l}\text { Hajili village with } 365 \text { peoples, } 1 \text { buffalo, } 2,4 \text { cows per a person, } \\
\text { average total biomass is } 17520 \mathrm{~kg} \text { on cattle-breeding }\end{array}$ & 61 & 16 & 39 \\
\hline 10 & $\begin{array}{l}\text { I Chagan village with } 365 \text { peoples, } 3,6 \text { buffalos, } 3,3 \text { cows per a } \\
\text { person, average total biomass is } 11251,6 \mathrm{~kg} \text { on cattle-breeding }\end{array}$ & 44 & 60 & 52 \\
\hline 11 & $\begin{array}{l}\text { II Chagan village with } 149 \text { peoples, } 4,3 \text { buffalos, } 4,7 \text { cows per a } \\
\text { person, average total biomass is } 8329,1 \mathrm{~kg} \text { on cattle-breeding }\end{array}$ & 62 & 71 & 67 \\
\hline 12 & $\begin{array}{l}\text { Kechmeddin village with } 211 \text { peoples, } 2,9 \text { buffalos, } 5,8 \text { cows per a } \\
\text { person, average total biomass is } 12132,5 \mathrm{~kg} \text { on cattle-breeding }\end{array}$ & 77 & 48 & 63 \\
\hline 13 & $\begin{array}{l}\text { Galaderesi village with } 143 \text { peoples, } 2,9 \text { buffalos, } 6,1 \text { cows per a } \\
\text { person, average total biomass is } 5634,2 \mathrm{~kg} \text { on cattle-breeding }\end{array}$ & 81 & 48 & 65 \\
\hline 14 & $\begin{array}{l}\text { Galeybugurd village with } 801 \text { peoples, } 2,6 \text { buffalos, } 5,2 \text { cows per a } \\
\text { person, average total biomass is } 19143,9 \mathrm{~kg} \text { on cattle-breeding }\end{array}$ & 69 & 43 & 56 \\
\hline 15 & $\begin{array}{l}\text { Pirbeyli village with } 1114 \text { peoples, } 4,4 \text { buffalos, } 3,8 \text { cows per a } \\
\text { person, average total biomass is } 25065 \mathrm{~kg} \text { on cattle-breeding }\end{array}$ & 50 & 73 & 61,5 \\
\hline 16 & $\begin{array}{l}\text { Gonagkend village with } 829 \text { peoples, } 3,4 \text { buffalos, } 4,9 \text { cows per a } \\
\text { person, average total biomass is } 25699 \mathrm{~kg} \text { on cattle-breeding }\end{array}$ & 65 & 56 & 60,5 \\
\hline 17 & $\begin{array}{l}\text { Gurdtepe village with } 804 \text { peoples, } 3,4 \text { buffalos, } 4,9 \text { cows per a } \\
\text { person, average total biomass is } 25245,6 \mathrm{~kg} \text { on cattle-breeding }\end{array}$ & 46 & 28 & 37 \\
\hline 18 & $\begin{array}{l}\text { Pirbeyli village with } 690 \text { peoples, } 1,2 \text { buffalos, } 2,4 \text { cows per a } \\
\text { person, average total biomass is } 24943,5 \mathrm{~kg} \text { on cattle-breeding }\end{array}$ & 32 & 20 & 26 \\
\hline 19 & $\begin{array}{l}\text { Malham village with } 192 \text { peoples, } 1,6 \text { buffalos, } 3,4 \text { cows per a } \\
\text { person, average total biomass is } 25404 \mathrm{~kg} \text { on cattle-breeding }\end{array}$ & 45 & 26 & 36 \\
\hline 20 & $\begin{array}{l}\text { Nagarakhana village with } 864 \text { peoples, } 1,7 \text { buffalos, } 3,3 \text { cows per a } \\
\text { person, average total biomass is } 48643,2 \mathrm{~kg} \text { on cattle-breeding }\end{array}$ & 44 & 28 & 36 \\
\hline 21 & $\begin{array}{l}\text { Pirbeyli village with } 184 \text { peoples, } 1,9 \text { buffalos, } 3,9 \text { cows per a } \\
\text { person, average total biomass is } 10837,6 \mathrm{~kg} \text { on cattle-breeding }\end{array}$ & 52 & 31 & 42 \\
\hline 22 & Pirgulu settlemnet & $\times$ & $\times$ & $\times$ \\
\hline 23 & $\begin{array}{c}\text { Safali village with } 134 \text { peoples, } 1 \text { buffalo, } 1,9 \text { cows per a person, } \\
\text { average total biomass is } 9366,6 \mathrm{~kg} \text { on cattle-breeding }\end{array}$ & 25 & 16 & 21 \\
\hline 24 & $\begin{array}{l}\text { Sis village with } 134 \text { peoples, } 1,9 \text { buffalos, } 3,9 \text { cows per a person, } \\
\text { average total biomass is } 23751 \mathrm{~kg} \text { on cattle-breeding }\end{array}$ & 52 & 31 & 42 \\
\hline 25 & $\begin{array}{l}\text { Talishnuru village with } 137 \text { peoples, } 1 \text { buffalo, } 1,9 \text { cows per a } \\
\text { person, average total biomass is } 14978,25 \mathrm{~kg} \text { on cattle-breeding }\end{array}$ & 25 & 16 & 21 \\
\hline 26 & $\begin{array}{l}\text { II Gizmeydan village with } 95 \text { peoples, } 1,5 \text { buffalos, } 3 \text { cows per a } \\
\text { person, average total biomass is } 4868,75 \mathrm{~kg} \text { on cattle-breeding }\end{array}$ & 40 & 25 & 33 \\
\hline 27 & $\begin{array}{l}\text { Pirbeyli village with } 233 \text { peoples, } 3,3 \text { buffalos, } 6 \text { cows per a person, } \\
\text { average total biomass is } 1217,19 \mathrm{~kg} \text { on cattle-breeding }\end{array}$ & 80 & 55 & 68 \\
\hline Sum: & 27 villages (included one settlement) & average & 39,5 & 46 \\
\hline
\end{tabular}


From the table it is cleared that in 31 villages including one settlement around Pirgulu region averagely total biomass amount obtained from cow and buffalo husbandries will be $562713,9+605307=1168021 \mathrm{~kg}$. Total capacity of biogas generated from the very biomass is $102278+60540=162818$ $\mathrm{m}^{3}$. in Pirgulu zone of Shamakhi region in 27 settlements in the personal cattle-breeding farms average daily biomass amount (by $20 \mathrm{~kg}$ biomass weight) from cow farm was calculated and determined. At the result $\left(1 \mathrm{~m}^{3}\right.$ biogas from $10 \mathrm{~kg}$ biomass) average annual and average monthly biogas generation, at the same time percentage index of total average monthly biogas potential in supplying gas demand was calculated.

Also by the new method average daily, annual biomass potential from buffalo farms (average weight is $25 \mathrm{~kg}$ ) and on this basis average annual and monthly biogas generation, then percentage index of biogas in total average monthly gas demand provision was calculated and defined.

Finally after having clarified biomass potential obtained from both cattle-breeding farms, it was revealed that biogas generation can provide the total gas demand averagely $(52+39,5) \div 2=46 \%$.

Also on the base of results the biomass potential possibilities have been determined for biogas generation. This gives opportunity to the investors to put capital for this energy field.

\section{Results and Discussions}

In the direction of renewable energy potential assessment and appreciation of Pirgulu region research was firstly realized. According to the monitoring, investigations and calculations carried out in 2004-2010 years in the region, beside solar and wind energy potentials of the zone biomass resource has been calculated. Total amount is enough for biogas generation in the villages surrounding Pirgulu region. All biomass has been obtained from cattle-breeding farms. Due to the reference analysis it is clear that none of the region of Azerbaijan including Pirgulu biomass potential for generating biogas hasn't implemented before. In settlements of investigation area several renewable energy potentials are to be utilized together in settlements located around woodla nds for supplying heat energy potential provision. In spite of solar and wind energy reserves biomass potential can be used in biogas generation. Personal cattle-breeding husbandries crate the suitable condition for collecting biomass reserves. This energy potential holds economical and ecological advantages for the population and the government. Because the biomass obtained from cattle farms is collected periodically and biogas generated from this biomass is able to supply the holder of that husbandry with gas for heating his house and farm. This is the irreplaceable energy source instead of fuelwood cut from the forests.

\section{Conclusions}

Calculations and observations have been realized in 31 settlements around the investigation area. Finally the the conclusions have been come into:

- Personal cattle-breeding consists of 4014,75 including 1338,25 buffalos and 2676,5 cows;

- In 31 settlments there are averagely 3,6 buffalos, 4,6 cows per a person, total amount is 3,9 from both;

- Average biomass amount due to the population biomass amount was determined $562713,9 \mathrm{~kg}$ from buffalo and $605307 \mathrm{~kg}$ from cow, total biomass from cattle-breeding farms is $1168020,9 \mathrm{~kg}$;

- Biogas generation consists averagly of $102278 \mathrm{~m}^{3}$ from buffalo biomass and $60540 \mathrm{~m}^{3}$ from cow biomass collected inthe personal husbandries, the total amount is $162818 \mathrm{~m}^{3}$.

It has been revealed on the reports realized in Pirgulu region since 2004 year, biomass energy potential holds great importance for protecting fuelwood from forests and supplyi ng energy need in the villages. There is enough biomass potential in the personal cattle-breeding husbandries.

\section{REFERENCES}

[1] Beer L.L., Boyd E.S., Peters J.W., Posewitz M.C. Engineering algae for biohydrogen and biofuel production Current Opinion in Biotechnology 20(3), 2009, pp. 264-271.

[2] Berndes G., Hoogwijk M., van den Broek R. The contribution of biomass in the future Bioenergy 20, 2001, pp. 151-159.

[3] Börjesson P., Ericsson K., Di Lucia L., Nilsson L.J., Åhman M. SUSTAINABLE VEHICLE FUELS - DO THEY EXIST? Department of Technology and Society, Lund University, Sweden, Report No. 67, 2009.

[4] Cleveland C.J., Kaufmann R., Stern S.I. Aggregation and the Role of Energy in the Economy. Ecological Economics 32, 2000, pp. 301-317.

[5] Dias R.A., Mattos C.R., Balestieri J.A.P. Energy education: breaking up the rational energy use barriers. Energy Policy 32(11), 2004, pp.1339-1347.

[6] Faaij A.P.C. 2008. "Sustainable Biofuels". Presentation at Roclfeller Bellagio Conference on North-South Biopact, 25 March, Bellagio, Italy. FAO. Global forest products consumption, production, trade and prices: global forest products model projections to 2010. United Nations Food Agricultural Organisation, Rome, Italy, 1998, p 345.

[7] Fischer G., Schrattenholzer L. Global bioenergy potentials through 2050. Biomass and Bioenergy 20, 2001, pp.151-159.

[8] Fujino J., Yamaji K., Yamamoto H. Biomass-balance table for evaluating bioenergy resources. Applied Energy 63(2), 1999, pp. 75-89.

[9] Global energy supply: a review of 17 studies. Biomass and Bioenergy 25(1), 2003, pp. 1-28.

[10] Hashiramoto O. Wood-product trade and policy issue. Cross -sectoral policydevelopments in forestry, 2007, pp.24-35.

[11] Mammadov F.F. Application of Solar Energy in the initial 
crude oil field. Journal of Energy in Southern Africa.vol 17, № 2, 2006, pp.27-30.

[12] Mammadov G.Sh. Azerbaijan Eco-ethic Problems. Baki: «Elm», 2004. 377 p.

[13] Mammadov G.Sh., Samadova U.F., Mammadov F.F. Ecological Protection of Forest Wood. Report of ANAS. 2009, pp. 27-30.

[14] Samadova U.F. Effective Protection of Forest Soils in Azerbaijan, International Journal of Academic Research, № 1, vol. 1, 2009, pp. 53-58.

[15] Samadova U.F. Importance of Solar Energy for the Environment. Municipality and Ecology: existing problems and their possible solution ways, Materials of Scientific Practical Conference. Baki, 2006, pp. 132-135.

[16] Samadova U.F. Solar and Wind Energy Application for the Woods and Forest Soil's Protection // Renewable Energy Congress X, Glasgow, Scotland, 19-25 July, 2008, pp. 2278-2280. 